\title{
Hybrid Modeling and Simulation of Biomolecular Networks
}

\author{
Rajeev Alur, Calin Belta, Franjo Ivančić, Vijay Kumar, Max Mintz, \\ George J. Pappas, Harvey Rubin, and Jonathan Schug \\ University of Pennsylvania \\ biohybrid@grasp.cis .upenn.edu
}

\begin{abstract}
In a biological cell, cellular functions and the genetic regulatory apparatus are implemented and controlled by a network of chemical reactions in which regulatory proteins can control genes that produce other regulators, which in turn control other genes. Further, the feedback pathways appear to incorporate switches that result in changes in the dynamic behavior of the cell. This paper describes a hybrid systems approach to modeling the intra-cellular network using continuous differential equations to model the feedback mechanisms and mode-switching to describe the changes in the underlying dynamics. We use two case studies to illustrate a modular approach to modeling such networks and describe the architectural and behavioral hierarchy in the underlying models. We describe these models using Charon [2], a language that allows formal description of hybrid systems. We provide preliminary simulation results that demonstrate how our approach can help biologists in their analysis of noisy genetic circuits. Finally we describe our agenda for future work that includes the development of models and simulation for stochastic hybrid systems 1
\end{abstract}

\section{Introduction}

In order to survive, organisms continuously monitor their surroundings and, if necessary, adjust traffic through simple or complex combinations of genetic and metabolic networks to respond to alterations in local conditions. Local conditions include both the physical environment, for example, temperature (the heat and cold shock response), nutrient and energy source concentrations (the stringent response), light (circadian rhythms), cell density (quorum sensing response) as well as the molecular environment of individual regulatory components. Examples of the latter include intracellular concentrations of transcription factors and allosteric effectors. The availability of complete genomic information for a wide variety of organisms and the consequent attention on proteomics has dramatically increased the number of systems and components of systems that are involved in these sensing and responding activities [410]. Understanding how

\footnotetext{
${ }^{1}$ This research was supported in part by DARPA/ITO Mobies project (grant number F33615-00-C-1707) and NSF grant CDS-97-03220.
} 
these biological systems are integrated and regulated and how the regulation may be influenced, possibly for therapeutic purposes, remains a significant challenge.

In this paper we model and simulate examples of genetic and metabolic networks using a hybrid systems approach that combines concepts and tools from control theory and computer science. First we analyze a previously published plasmid-based genetic network that was designed and synthesized using three repressor transcription factors where one repressor negatively regulates the production of a subsequent repressor [7. Then we model a biologically important genetic network that controls the quorum sensing response, an adaptive response of certain gram negative bacteria to local population density [1317. The quorum sensing response controls the luminescent behavior in certain strains of Vibrio which has been linked to the normal development of the bacterial host [18] as well as to medically important phenomena such as biofilm formation by Pseudomonas aerugenosa, an organism that can cause overwhelming pneumonia and septic shock [1120].

\section{Modeling}

The genetic circuits and biomolecular networks considered here and elsewhere are remarkably similar to hybrid systems encountered in engineering, for example embedded systems. In particular, it is worth noting the following three key features:

Concurrency and communication. At the intra-cellular level, proteins and mRNAs are agents communicating with each other and influencing each other's behavior. At the inter-cellular level, cells can be viewed as networked agents interacting with each other via different communication mechanisms.

Discrete and continuous behaviors. At the lowest level, the evolution of entities such as proteins can be described by differential equations. Discreteness arises in two ways. First, a certain activity may be triggered only when the concentration of enabling quantities is above the desired threshold. This leads to discrete switching between active and dormant states. Second, different models may be appropriate at different levels of concentration.

Stochastic behavior. Evolution of entities is not deterministic, and is better captured by stochastic models that allow for uncertainty and noise.

These characteristics are typical of high-level models of embedded software such as autonomous communicating mobile robots. For describing such systems, we have developed the language CHARON [2] which incorporates ideas from concurrency theory (languages such as CSP [12]), object-oriented software design notations (such as Statecharts 9] and UML [3]), and formal models for hybrid systems (such as hybrid automata [1] and hybrid I/O automata [15]). The key features of CHARON are:

Architectural hierarchy. The building block for describing the system architecture is an agent that communicates with its environment via shared 
variables. The language supports the operations of composition of agents to model concurrency, hiding of variables to restrict sharing of information, and instantiation of agents to support reuse.

Behavior hierarchy. The building block for describing flow of control inside an atomic agent is a mode. A mode is basically a hierarchical state machine, that is, a mode can have submodes and transitions connecting them. Variables can be declared locally inside any mode with standard scoping rules for visibility. Modes can be connected to each other only via well-defined entry and exit points. We allow sharing of modes so that the same mode definition can be instantiated in multiple contexts. Finally, to support exceptions, the language allows group transitions from default exit points that are applicable to all enclosing modes.

Discrete updates. Discrete updates are specified by guarded actions labeling transitions connecting the modes. Actions can have calls to externally defined Java functions which can be used to write complex data manipulations. It also allows us to mimic stochastic aspects through randomization.

Continuous updates. Some of the variables in CHARON can be declared ana$\log$, and they flow continuously during continuous updates that model passage of time. The evolution of analog variables can be constrained in three ways: differential constraints (e.g. by equations such as $\dot{x}=f(x, u)$ ), algebraic constraints (e.g. by equations such as $y=g(x, u)$ ), and invariants (e.g. $|x-y| \leq \varepsilon$ ) which limit the allowed durations of flows. Such constraints can be declared at different levels of the mode hierarchy.

Modular features of CHARON allow succinct and structured description of complex systems. Similar features are supported by the languages SHIFT [6] and STATEFLOW (see www.mathworks.com). In CHARON, modularity is not only apparent in syntax, but we are developing analysis tools (such as simulation) that exploit this modularity. Furthermore, CHARON has formal foundations supporting compositional refinement calculus which allows relating different models of the system in mathematically precise manner. A formal mathematical description allows us to develop tools for computing equilibria, for reachability analysis and for analyzing properties like stability and reachability.

In the next two sections, we will briefly describe case studies that we have used to investigate the hybrid systems approach to modeling biological systems, and the applications of CHARON to these systems. We will also illustrate our approach by providing preliminary simulation results.

\section{A Repressilator Network}

As noted in [5], most biomolecular systems of interest involve many interactions connected through positive and negative feedback loops and an understanding of their dynamics is hard to obtain. In this section we will describe the modeling of a specific biomolecular network. We will model a repressilator system described in [7]. First we provide some biological background information and describe the 
protein network used in [7], and then describe the models of the protein network, including examples of CHARON models.2

\subsection{The Basic Phenomena}

In the synthetic oscillatory network described in [7, networks of interacting biomolecules carry out many essential functions in living cells. But the design principles of the functioning of such networks still remain poorly understoodeven in relatively simple systems [14. The authors proposed the design and construction of a synthetic protein network implementing a particular function. Their motivation is that such "rational network design" may lead to the engineering of new cellular behaviors and to improved understanding of naturally occuring networks.

The repressilator system described in [7] contains three proteins, namely lacI, tetR, and cI. The protein lacI represses the protein tetR, tetR represses cI, whereas cI represses lacI, thus completing a feedback system called a repressilator system. The dynamics of the network depend on the transcription rates, translation rates, and decay rates of proteins and messenger RNAs. Depending on the values of the different parameters in the model, the system might converge to a stable limit cycle or become unstable.

\subsection{Approaches to Modeling}

It is well known in mechanics and thermodynamics that there are two different approaches to modeling systems such as the repressilator system. At reasonably high molecular concentrations, one can adopt continuum models which lend themselves to deterministic models involving ordinary and partial differential equations. At lower concentrations, the discrete molecular interactions become important and deterministic models are difficult to obtain [8].

The Deterministic, Continuous Approximation. We will consider the three repressor protein concentrations $p_{i}, i \in P=\{$ lacI, tetR, $\mathrm{cI}\}$ and their corresponding mRNA concentrations $m_{i}, i \in P$ as continuous dynamic variables. The system kinetics are determined by the following six coupled first-order differential equations.

$$
\begin{gathered}
\frac{d m_{i}}{d t}=-m_{i}+\frac{\alpha}{1+p_{j}^{n}}+\alpha_{0} \\
\frac{d p_{i}}{d t}=-\beta\left(p_{i}-m_{i}\right) \\
(i, j) \in\{(\text { lacI }, \mathrm{cI}),(\text { tetR,lacI }),(\mathrm{cI}, \text { tetR })\}
\end{gathered}
$$

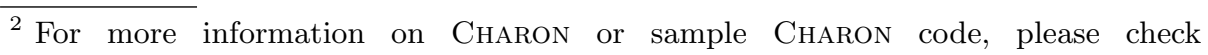
http://www.cis.upenn.edu/mobies/charon/ or contact ivancic@seas.upenn.edu. 
The equations use various constants. The leakiness of the promoter $\alpha$ is the number of protein copies per cell produced from a given promoter type during continuous growth in the presence of saturating repressor amounts. During the absence of the repressor, we have $\alpha+\alpha_{0}$ number of protein copies per cell. The ratio of the protein decay rate to the mRNA decay rate is denoted by $\beta$, while $n$ stands for the so called Hill coefficient.

The Stochastic, Discrete Approximation. The continuous analysis neglects the discrete nature of molecular components and the stochastic character of their interaction [7]. Following [7], we adopt the stochastic approximation as described by Gillespie in [8]. The various proteins and mRNAs are modeled by discrete variables corresponding to the number of molecules measuring concentration, and are updated at discrete time intervals by stochastic rules.

\subsection{Charon Model}

In this section we will present the repressilator system models as described in [7] using the CHARON language. We will present many of the advantages that the Charon language has to offer for modeling such biomolecular models.

Our model will define a generic protein model as an agent in CHARON. We will instantiate this agent model to obtain the three proteins lacI, tetR, and cI. The approximation models will be implemented inside the modes of the protein agent. To present another feature of our language, we will also describe a combination of the discrete and the continuous model into one modeling system.

The Protein Agent in the Continuous Approximation. In this section we will describe a CHARON model of a generic protein agent. We have a continuous input variable which represents the repressor protein concentration $p_{R}$. This means, that the environment of this protein agent supplies the value of this variable, and it cannot be changed by the protein agent. The protein agent has a continuous private variable representing the messenger RNA concentration. Private variables cannot be seen outside the agent and can be updated internally for internal use only. The output of the protein agent is a continuous variable representing the protein concentration. Output variables are updated by the agent, and can be used as input variables to other agents. The generic protein agent has parameters $\alpha_{0}, \alpha, \beta, n, p_{0}$, and $m_{0}$. By instantiating these parameters with values, we can obtain instantiated protein agents representing a specific protein. The parameters $p_{0}$ and $m_{0}$ will be used for initialization purposes and stand for the initial protein concentration and the initial messenger RNA concentration respectively. The following represents the corresponding CHARON code.

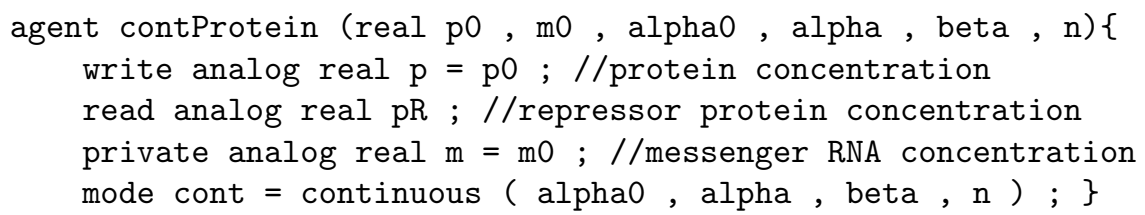




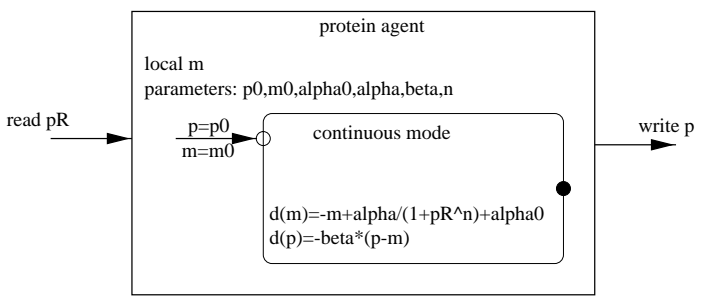

Fig. 1. A generic protein agent for the continuous approximation model

We still need to define the behavior of the agent. The behavior is described by the modes of the agent. The behavior of the generic protein agent is defined in cont, which is an instantiation of a generic continuous mode defined by the following code. A graphical version of the generic protein model can be found in Figure 1.
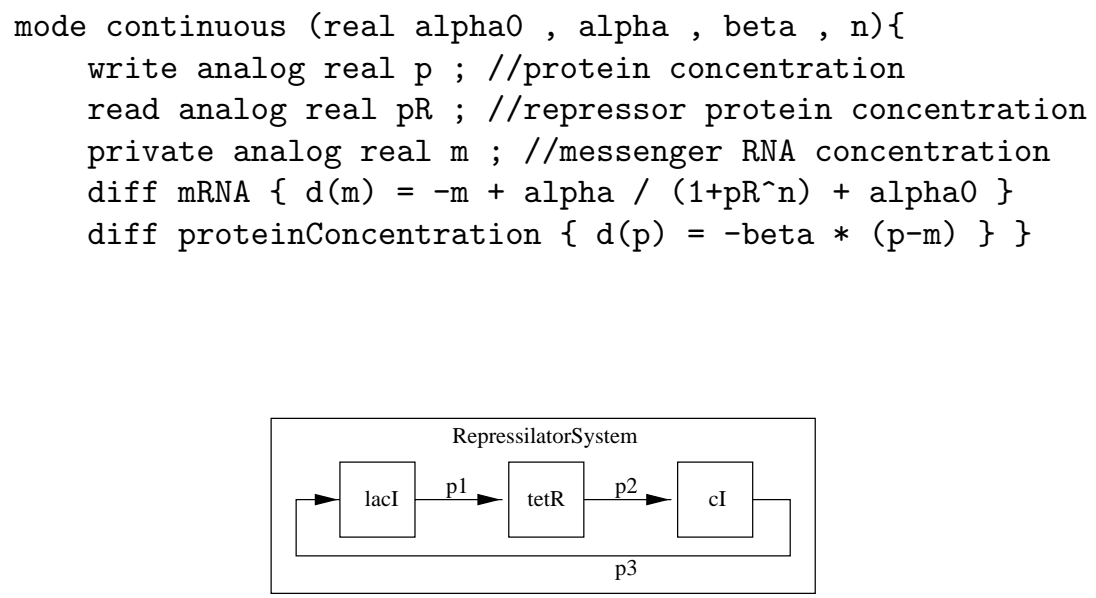

Fig. 2. Composed repressilator system using the instantiated generic protein agent

Instantiation and Concurrency. We defined a generic protein agent in the previous section. We have to instantiate this generic agent model to get the three proteins used in the system. We also want the three proteins lacI, tetR, and cI to run in parallel and to influence each other. Notice the use of renaming of variables to couple the three instantiated protein agents to influence each other. A graphical version of the composed system is illustrated in Figure 2] The following represents the corresponding CHARON code using some values for the parameters. A simulation trace generated by the CHARON tool-set is given in Figure 3. 

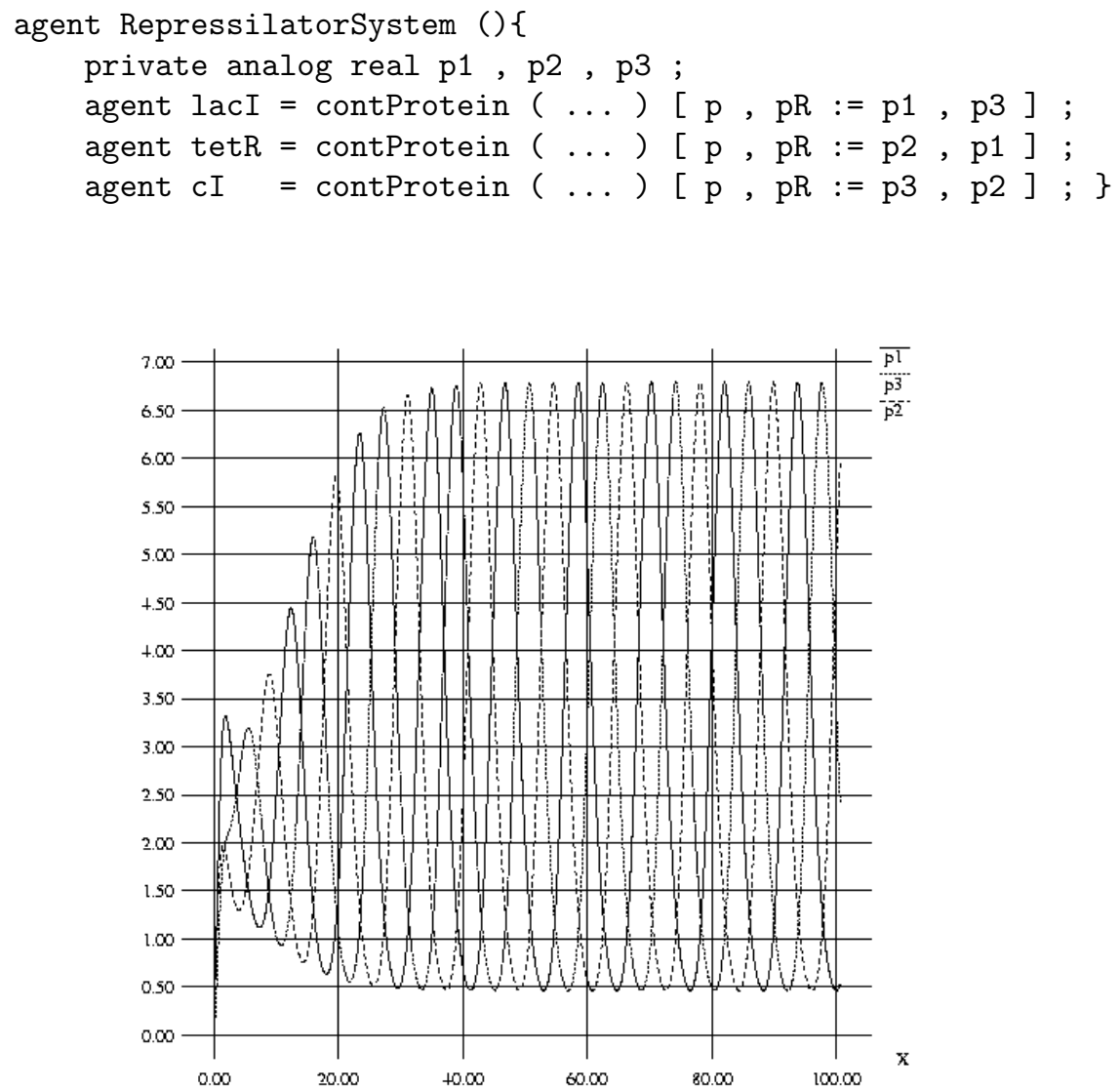

Fig. 3. Simulation trace for the repressilator system showing stable oscillations for the three protein concentration $p_{1}, p_{2}, p_{3}$ over time.

The Protein Agent in the Discrete Approximation. In this section we will present a possible model for a discrete approximation of a protein agent. As we did it for the continuous case, we will again define a generic protein agent, that can be instantiated to build a system of proteins. Our model works as follows. We have an integer variable $n$ that keeps track of the number of protein molecules which is the output of the agent. The input to the agent is the number of repressor protein molecules $n_{R}$. Depending on various parameters, we want to increase or decrease the number of protein molecules by one at a time. The basic idea is to use stochastic simulation as described in [8]. The parameters that influence the stochastic simulation are binding and unbinding of proteins on two-sided promoters, the protein and mRNA decay rates, and translation. 


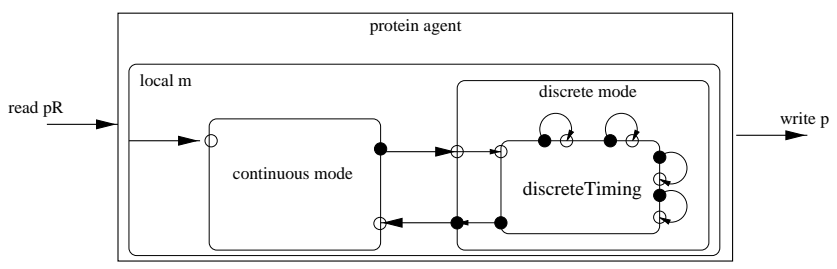

Fig. 4. A generic protein agent for the combined framework using continuous and discrete approximation model

Combining the two Models into one Framework. The two different models for the repressilator system can be combined into one framework. The basic idea is to use the deterministic continuous model whenever the concentration of the protein is high enough, whereas we would switch to the discrete, stochastic model if the concentration would fall below a certain threshold value. Figure 4 gives an intuitive graphical representation of the protein agent with both the continuous and discrete approximation.

\section{Quorum Sensing in Bacteria}

A good illustration of multicellular behavior in prokaryotes is the cell-densitydependent gene expression. In this process, a single cell is able to sense when a quorum of bacteria, a minimum population unit, is achieved. Under these conditions, certain behavior is efficiently performed by the quorum, such as bioluminescence, which is the best known model for understanding the mechanism of cell-density-dependent gene expression. In this section, we will describe a hybrid system model that captures the changes in dynamics of the biochemical reactions observed in the literature [13 16 17].

\subsection{The Basic Phenomena}

Vibrio fischeri is a marine bacterium that can be found both as a free-living organism and as a symbiont of some marine fish and squid. As a free-living organism, V. fisheri exists at low densities (less than 500 cells per ml of seawater) and appears to be non-luminescent. As a symbiont, the bacteria live at high densities and are, usually, luminescent. In a liquid culture, the bacteria's level of luminescence is low until the culture reaches mid to late exponential phase. A dramatic increase in luminescence is observed at that time due to the transcriptional activation of the lux genes. Once the bacteria reach stationary phase, the level of luminescence decreases.

The lux regulon [17] contains two operons, $O_{L}$ and $O_{R}$ (see Figure 5). The left operon $O_{L}$ contains the $\operatorname{lux} R$ gene encoding the protein LuxR, a transcriptional activator of the system. The right operon $O_{R}$ contains seven genes luxICDABEG. 


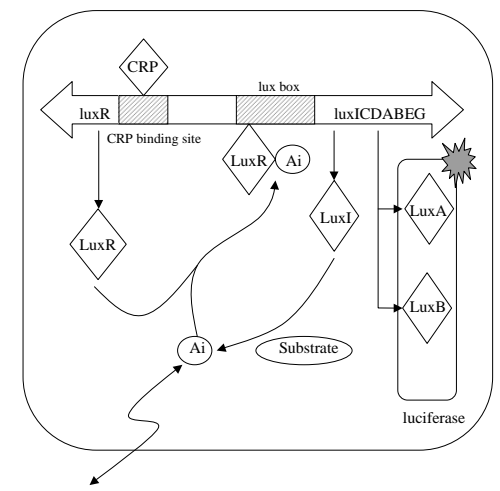

Fig. 5. A portion of DNA emphasizing luxR and luxICDABEG genes and the binding sites for LuxR complex and CRP

Protein LuxI, the product of the luxI gene is required for endogenous production of autoinducer, a small molecule capable of diffusing in and out of the cell membrane. Genes $\operatorname{lux} A$ and $\operatorname{lux} B$ encode two subunits of luciferase. The trio $\operatorname{lux} C$, luxD, and luxE code for the subunits of a protein complex which provides an aldehyde substrate for luciferase. The function of $\operatorname{lux} G$ is unknown. The autoinducer Ai binds to protein LuxR to form a complex Co. The two operons are separated by a regulatory region that contains a binding site for the cyclic AMP receptor protein $\mathrm{CRP}$ and a binding site for the complex Co.

The transcription of $l u x R$ is regulated by both CRP and Co. We can distinguish among the following three different cases:

- Case $O_{L}-1$ In the absence of the autoinducer, CRP activates $O_{L}$ expression by initiating two RNA transcripts.

- Case $O_{L}-2$ At low autoinducer concentrations, luxR transcription is stimulated by increasing CRP-dependent transcription and by Co-dependent transcription from another transcriptional start site.

- Case $O_{L} \mathbf{- 3}$ At high autoinducer concentrations, lux $R$ transcription is repressed through a second, weaker Co binding site located in luxD.

Likewise, transcription of $O_{R}$ is regulated by both CRP and Co. We distinguish two different cases:

- Case $O_{R}$-1 In the absence of autoinducer, CRP represses $O_{R}$ transcription.

- Case $O_{R}-2$ In the presence of autoinducer, Co activates transcription of $O_{R}$.

These cases will be interpreted as modes as seen later in the paper.

\subsection{Mathematical Model}

In this section, we develop a mathematical model for the luminescence phenomenon in one bacterium of $V$. fischeri, describing the concentrations of the 
relevant mRNA's, proteins, and small molecules. As described in Section 4.1 the mechanism of transcription activation of both operons is highly dependent on the concentration of autoinducer, so the time evolution of the system cannot be described by one set of continuous differential equations 3 Combining cases for $O_{L}$ and $O_{R}$ given in the previous section, yields three modes, which we call $O F F$, $P O S$ and NEG. The transitions between modes are governed by the level of internal autoinducer which we represent by [Ai]. Mode $O F F$ corresponds to very low or zero concentration of autoinducer $\left([\mathrm{Ai}] \leq[\mathrm{Ai}]_{-}\right)$within the bacterium and no luminescence is observed. The system is in mode POS when the concentration of internal autoinducer is low $\left([\mathrm{Ai}]_{-} \leq[\mathrm{Ai}] \leq[\mathrm{Ai}]_{+}\right)$. This mode corresponds to positive growth and increasing concentration of autoinducer. Luminescence is observed, as are higher concentrations of proteins LuxA, LuxB, LuxC, LuxD, and LuxE. The transition to mode $N E G$ (negative growth) occurs at high levels of autoinducer $\left([\mathrm{Ai}]>[\mathrm{Ai}]_{+}\right)$.

We use the following rate equation to describe the concentration for any molecular species (mRNA, protein, protein complex, or small molecule) [19]:

$$
\frac{d[x]}{d t}=\text { synthesis }- \text { decay } \pm \text { transformation } \pm \text { transport }
$$

The synthesis term represents transcription for mRNA and translation for proteins. The decay term represents a first order degradation process. The transformation term describes reactions such as cleavage or ligand-binding that do not destroy the protein, but do remove its ability to participate in specific reactions. Finally, molecular species may participate in transport processes, like passive diffusion or active transport through a membrane.

The biomolecular system can be described in a nine dimensional state space. The nine variables, $x_{1}, x_{2}, \ldots, x_{9}$, describe the concentrations of different molecules as follows:

$$
\begin{aligned}
& x_{1}=\text { mRNA transcribed from } O_{L}, \\
& x_{2}=\text { mRNA transcribed from } O_{R}, \\
& x_{3}=\text { protein LuxR, } \\
& x_{4}=\text { protein LuxI, } \\
& x_{5}=\text { protein LuxA, } \\
& x_{6}=\text { protein LuxB, } \\
& x_{7}=\text { autoinducer inside the bacterium } \mathrm{Ai}, \\
& x_{8}=\text { LuxR:Ai complex Co, } \\
& x_{9}=\text { autoinducer outside the bacterium } \mathrm{Ai}_{e x}, \\
& \text { where } \mathrm{Ai} \text { is the dimensionless version of }[\mathrm{Ai}] .
\end{aligned}
$$

For simplicity, we have assumed that the concentrations of CRP and of the substrate necessary for endogenous production of $\mathrm{Ai}$ are constant. Further, we have neglected the decay rates for chemical compounds. Finally, we assume that

\footnotetext{
${ }^{3}$ In [13], the differential equations for the low autoinducer concentration are described. The model presented here describes a wider range of operating conditions.
} 
the concentrations of LuxC, LuxD, and LuxE are similar to those of LuxA and LuxB.

The (continuous) differential equations for each mode are of the form $\dot{x}=f^{i}(x)$ where $x=\left[x_{1}, x_{2}, \ldots, x_{9}\right]^{T} \in \mathbb{R}^{9}, f^{i}=\left[f_{1}^{i}, f_{2}^{i}, \ldots, f_{9}^{i}\right]$, and $i \in\{O F F, P O S, N E G\}$. The components of the vector fields are explicitly given by:

$$
\begin{aligned}
f_{1}^{O F F} & =\eta_{1}\left(\frac{1}{2} c-x_{1}\right) \\
f_{1}^{P O S} & =\frac{\eta_{1}}{4}\left(3 c+\frac{x_{8}^{\nu_{81}}}{\kappa_{81}^{\nu 81}+x_{8}^{\nu 81}}-4 x_{1}\right) \\
f_{1}^{N E G} & =-\eta_{1} x_{1} \\
f_{2}^{O F F} & =-\eta_{2} x_{2} \\
f_{2}^{P O S}=f_{2}^{N E G} & =\eta_{2}\left(\frac{x_{8}^{\nu 8}}{\kappa_{82}^{\nu 82}+x_{8}^{\nu 8}}-x_{2}\right) \\
f_{3}^{i} & =\eta_{3}\left(x_{1}-x_{3}\right)-r_{37}, A i x_{3} x_{7} \\
f_{4}^{i} & =\eta_{4}\left(x_{2}-x_{4}\right)-r_{4} x_{4} \\
f_{5}^{i} & =\eta_{5}\left(x_{2}-x_{5}\right) \\
f_{6}^{i} & =\eta_{6}\left(x_{2}-x_{6}\right) \\
f_{7}^{i} & =-\eta_{7} x_{7}+r_{4} x_{4}-r_{m e m}\left(x_{7}-x_{9}\right)-r_{37, R} x_{3} x_{7} \\
f_{8}^{i} & =-\eta_{8} x_{8}+r_{37, A i} x_{3} x_{7} \\
f_{9}^{i} & =-\eta_{7} x_{9}+r_{m e m}\left(x_{7}-x_{9}\right)+u
\end{aligned}
$$

where, in the last seven equations $f_{j}^{i}$ is independent of the mode. All the quantities in the above model are non-dimensional. $\eta_{i}=T_{0} / H_{i}$ where $T_{0}$ is the characteristic time constant of the system and $H_{i}$ is the half-life (inverse of the decay rate) of molecule $x_{i} . \nu_{i j}$ is a cooperativity coefficient while $\kappa_{i j}$ describes the potency of the regulation of the transcription of mRNA $j$ by protein $i$. $r$ denotes transformation and transfer rates. For example $r_{m e m}$ is the transfer rate of autoinducer through the membrane of the cell while $r_{37, R}$ and $r_{37, A i}$ are transformation rates obtained by non-dimensionalizing the binding rate of the reaction between $\mathrm{Ai}$ and LuxR in two different ways. $c$ is dependent on the concentration of CRP and its affinity to the corresponding binding site, and, as stated earlier, is assumed to be constant. Finally, $u$ emulates an external source of Ai and is used to simulate the sensitivity of the bacterium to changes of autoinducer concentration in the exterior.

We regard $u$ as an input to our system. Since proteins LuxA and LuxB are subunits of luciferase, which produces luminescence, it is reasonable to assume that the level of luminescence is proportional to the product of the concentrations of LuxA and LuxB, which we choose to be the output of the system.

\subsection{Charon Model}

The behavioral hierarchy in CHARON (see Figure 6) is characterized by three different behaviors which are represented by three different modes, namely $O F F$, 
$P O S$, and NEG. Many of the differential equations governing the dynamics of the system are shared between the modes. We will introduce the notion of mode hierarchy to extract the shared constraints. Through the notion of submodes and scoping, we can simplify the description of the respective modes $O F F, P O S$, and $N E G$.

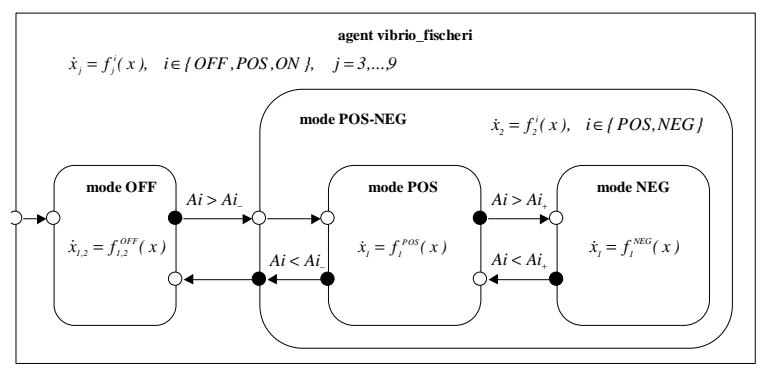

Fig. 6. ChARON structure of the system

Figure 7 illustrates the response (i.e., luminescence) of the bacterium to a perturbation in the concentration of external autoinducer that takes the form of a rectangular pulse. The magnitude of the step has been chosen to make the system go through all three modes. The results confirm the experimental observations [17): luminescence increases during mode POS and decreases in mode $N E G$; there is no luminescence in mode $O F F$. The switch history and the time evolution of the concentrations of the significant molecules in the system are also shown. In mode $O F F$, all molecules decay to zero, except for mRNA $O_{L}$ and the corresponding protein $R$, as expected. For a short time, in mode $P O S$, all the concentrations increase until the internal autoinducer reaches a high concentration, when the system is switched to mode $N E G$. In this last mode, everything decays to zero, except for internal autoinducer which can reach a stable non-zero value dependent on the size of the step of external autoinducer.

\section{Conclusions}

In this paper we have shown that biological cellular networks can be naturally modeled as hybrid systems. In particular, the protein repressilator system switches between a continuous deterministic model at high concentrations, and a timed, discrete, stochastic model at low concentrations. Similarly, the luminescence control of Vibrio fischeri is naturally modeled as a multi-modal hybrid system, resulting in simulations that are in accordance with experimental observations. The hybrid nature of such protein networks can be very easily expressed and simulated in CHARON, which may offer us better and a more global understanding of biological networks. 

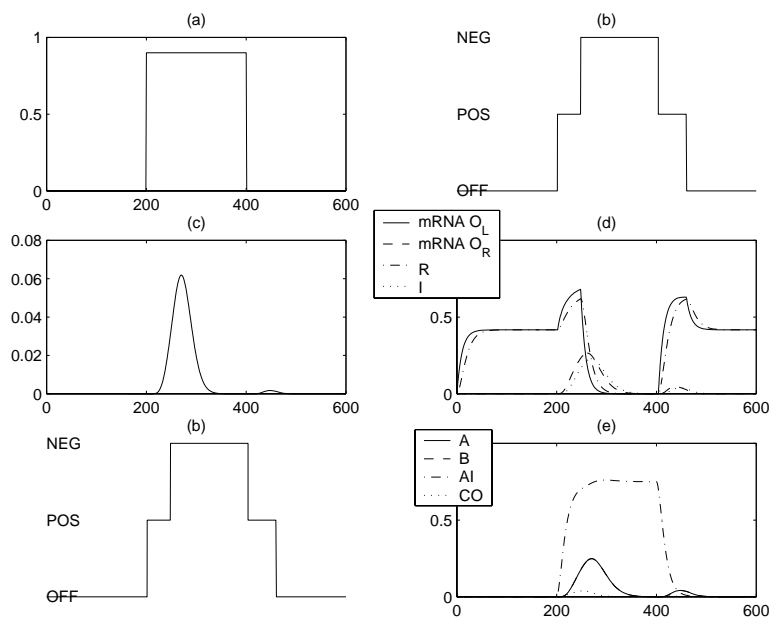

Fig. 7. Increase in external autoinducer produces luminescence: (a)input - external source of autoinducer; (b) switch history; (c) output (luminescence)- product of concentrations of proteins $A$ and $B$; (d) and (e) time - evolution of concentrations;

The enormous complexity of large scale biological networks will present us with great challenges that we must face. Exploiting the structure of biological systems will be critical for scaling the applicability of the modeling, analysis, and simulation tools. It is therefore extremely encouraging that the two case studies presented in this paper exhibit the architectural paradigms of modern software engineering.

We envision the link between hybrid systems technology, and biology to strengthen. The scalable nature of computational tools like CHARON will enable the unified and improved modeling of biological cellular networks, leading to better understanding, as well as providing us with the opportunity to determine how local biological changes can affect global behavior. Conversely, a good understanding of the robustness of noisy biological networks will lead to new approaches to designing networked embedded systems.

The case studies also highlight the need for developing a theory of stochastic hybrid systems, for instance, for modeling rate equations of biochemical reactions. We believe that mathematical and computational tools for the analysis of such systems present a research challenge for the hybrid systems community, while presenting a significant potential for greatly impacting post genomics research.

\section{References}

1. R. Alur, C. Courcoubetis, N. Halbwachs, T.A. Henzinger, P. Ho, X. Nicollin, A. Olivero, J. Sifakis, and S. Yovine. The algorithmic analysis of hybrid systems. Theoretical Computer Science, 138:3-34, 1995. 
2. R. Alur, R. Grosu, Y. Hur, V. Kumar, and I. Lee. Modular specifications of hybrid systems in CHARon In Hybrid Systems: Computation and Control, Third International Workshop, volume LNCS 1790, pages 6-19, 2000.

3. G. Booch, I. Jacobson, and J. Rumbaugh. Unified Modeling Language User Guide. Addison Wesley, 1997.

4. R. Brent. Genomic biology. Cell, 100(1):169-183, January 2000.

5. H. de Jong, M. Page, C. Hernandez, H. Geiselmann, and S. Maza. Modeling and Simulation of Genetic Regulatory Networks. ERCIM News, 43, October 2000.

6. A. Deshpande, A. Göllu, and L. Semenzato. SHIFT programming language and run-time systems for dynamic networks of hybrid automata. Technical report, University of California at Berkeley, 1997.

7. M. Elowitz and S. Leibler. Asynthetic oscillatory network of transciptional regulators. Nature, 403:335-338, January 2000.

8. D.T. Gillespie. Exact stochastic simulation of coupled chemical reactions. J. Phys. Chem., 81:2340-2361, 1977.

9. D. Harel. Statecharts: A visual formalism for complex systems. Science of Computer Programming, 8:231-274, 1987.

10. L.H. Hartwell, J.J. Hopfield, S. Leibler, and A.W. Murray. From molecular to modular cell biology. Nature, 402((6761 Suppl)):C47-52, December 1999.

11. D.J. Hassett, J.F. Ma, J.G. Elkins, T.R. McDermott, U.A. Ochsner, S.E. West, C.T. Huand, J. Fredericks, S. Burnett, P.S. Stewart, G. McFeters, L. Passador, and B.H. Iglewski. Quorum sensing in Pseudomonas aeruginosa controls expression of catalase and superoxide dismutase genes and mediates biofilm susceptibility to hydrogen peroxide. Mol Microbiol, 34(5):1082-1093, December 1999.

12. C.A.R. Hoare. Communicating Sequential Processes. Prentice-Hall, 1985.

13. S. James, P. Nilson, G. James, S. Kjellenberg, and T. Fagerstrom. Luminescence control in the marine bacterium Vibrio fischeri: An analysis of the dynamics of lux regulation. J Mol Biol, 296(4):1127-1137, March 2000.

14. D.E. Jr Koshland. The era of pathway quantification. Science, 280:852-853, 1998.

15. N. Lynch, R. Segala, F. Vaandrager, and H. Weinberg. Hybrid I/O automata. In Hybrid Systems III: Verification and Control, LNCS 1066, pages 496-510, 1996.

16. H. H. McAdams and A. Arkin. Simulation of prokaryotic genetic circuits. Annu. Rev. Biophys. Biomol. Struct., 27:199-224, 1998.

17. D.M. Sitnikov, J.B. Schineller and T.O. Baldwin. Transcriptional regulation of bioluminesence genes from Vibrio fischeri. Mol Microbiol, 17(5):801-812, September 1995.

18. K.L. Visick, J. Foster, J. Doino, M. McFall-Ngai and E.G. Ruby. Vibrio fischeri lux genes play an important role in colonization and development of the host light organ. Bacteriol, 182(16):4578-4586, August 2000.

19. G. von Dassow, E. Meir, E. M. Munro, and G. M. Odell. The segment polarity network is a robust development module. Nature, 406:188-192, July 2000.

20. H. Yang, M. Matewish, I. Loubens, D.G. Storey, J.S. Lam, and S. Jin. migA, a quorum-responsive gene of Pseudomonas aeruginosa, is highly expressed in the cystic fibrosis lung environment and modifies low-molecular-mass lipopolysaccharide. Microbiology, 146((Pt 10)):2509-2519, October 2000. 